\title{
Effects of quenched impurities on surface diffusion, spreading, and ordering of
} O/W(110)

Nikunen, P.; Vattulainen, Ilpo Tapio; Ala-Nissila, T.

Published in:

Journal of Chemical Physics

Link to article, DOI:

$10.1063 / 1.1505856$

Publication date:

2002

Document Version

Publisher's PDF, also known as Version of record

Link back to DTU Orbit

Citation (APA):

Nikunen, P., Vattulainen, I. T., \& Ala-Nissila, T. (2002). Effects of quenched impurities on surface diffusion, spreading, and ordering of O/W(110). Journal of Chemical Physics, 117(14), 6757-6765.

https://doi.org/10.1063/1.1505856

\section{General rights}

Copyright and moral rights for the publications made accessible in the public portal are retained by the authors and/or other copyright owners and it is a condition of accessing publications that users recognise and abide by the legal requirements associated with these rights.

- Users may download and print one copy of any publication from the public portal for the purpose of private study or research.

- You may not further distribute the material or use it for any profit-making activity or commercial gain

- You may freely distribute the URL identifying the publication in the public portal

If you believe that this document breaches copyright please contact us providing details, and we will remove access to the work immediately and investigate your claim. 


\title{
Effects of quenched impurities on surface diffusion, spreading, and ordering of $\mathrm{O} / \mathrm{W}(110)$
}

\author{
P. Nikunen \\ Helsinki Institute of Physics and Laboratory of Physics, Helsinki University of Technology, \\ P.O. Box 1100, FIN-02015 HUT, Espoo, Finland and Laboratory of Computational Engineering, \\ Helsinki University of Technology, P.O. Box 9400, FIN-02015 HUT, Finland
}

I. Vattulainen ${ }^{\text {a) }}$

Department of Chemistry, Technical University of Denmark, Building 207, DK-2800 Lyngby, Denmark and Laboratory of Physics and Helsinki Institute of Physics, Helsinki University of Technology, P.O. Box 1100, FIN-02015 HUT, Espoo, Finland

\author{
T. Ala-Nissila \\ Helsinki Institute of Physics and Laboratory of Physics, Helsinki University of Technology, \\ P.O. Box 1100, FIN-02015 HUT, Espoo, Finland and Department of Physics, Brown University, Providence, \\ Rhode Island 02912-1843
}

(Received 21 December 2001; accepted 17 July 2002)

\begin{abstract}
We study how quenched impurities affect the surface diffusion and ordering of strongly interacting adsorbate atoms on surfaces. To this end, we carry out Monte Carlo simulations for a lattice-gas model of $\mathrm{O} / \mathrm{W}(110)$, including small concentrations of immobile impurities which block their adsorption sites. We examine the behavior of the diffusion coefficients and order parameters as a function of coverage corresponding to various ordered phases at low temperatures. The effects of impurities are examined under both equilibrium and nonequilibrium conditions, and the results are compared to recent studies on a completely clean surface. We find that even minute impurity concentrations affect the diffusion behavior considerably in equilibrium. The effects are strongest in ordered phases and close to phase boundaries, where quenched impurities lead to a reduction of order, which in turn leads to significant changes in the collective diffusion and phase behavior. As the impurity concentration is increased to a level of a few percent of the total surface area, the reduction in order becomes particularly prominent at high coverages. Further studies under nonequilibrium conditions reveal that nonequilibrium effects are strong in the absence of impurities, while for surfaces covered by impurities the nonequilibrium effects are relatively weaker. (c) 2002 American Institute of Physics. [DOI: 10.1063/1.1505856]
\end{abstract}

\section{INTRODUCTION}

Surfaces play a key role in various important technological applications such as the growth of semiconductor structures for electronic devices, the purification of exhaust gases in the automotive industry, and the wetting of solid materials by liquids to reduce interfacial tension. ${ }^{1-3}$ In many cases, surface diffusion plays a prominent role and may even constitute the rate limiting step in the process. Therefore, more and more effort has been directed toward understanding the physical laws of nature that govern the diffusion of adatoms and more complex molecules on solid surfaces.

In this respect, the last few decades have been very successful. Thanks to a wide range of experimental techniques ${ }^{3-7}$ such as scanning tunneling microscopy and field ion microscopy, knowledge of surface structures and related diffusion mechanisms ${ }^{7-10}$ is nowadays reasonably good. Experimental works have been complemented by various theoretical activities, ${ }^{11-13}$ which in turn have provided plenty of insight into the understanding of the microscopic

\footnotetext{
a) Author to whom correspondence should be addressed. Electronic mail: Ilpo.Vattulainen@csc.fi.
}

details of diffusion processes on solid surfaces. Due to all these activities, it is fair to say that many of the prominent features of surface diffusion under ideal conditions in equilibrium are now well understood.

The situation becomes much more complicated, however, when the system is no longer ideal, but contains impurities, steps, or other defects that are typical under realistic conditions. ${ }^{4}$ The role of impurities, in particular, can be very dramatic as regards the growth of surfaces or the diffusion of adparticles on surfaces. This has been observed in various cases as regards the diffusion of individual (tracer) particles, in which case many of the generic features due to impurities and defects are known reasonably well (see below). As becomes evident in the discussion below and which is worthwhile to emphasize already at this stage, the effects due to impurities and defects on the collective aspects of surface systems are much more poorly understood. This is rather surprising since a number of phenomena including ordering and collective diffusion of surface systems are governed by the collective nature of many-body systems, which impurities and defects are expected to perturb. To demonstrate the role of impurities in surface diffusion and, in particular, to emphasize how the collective aspects of diffusion may be 
distinctly different from the better known case of singleparticle (tracer) diffusion, we therefore wish to discuss the two cases separately.

First, as far as single-particle diffusion is concerned, there is ample evidence that impurities and other defects play a major role in related processes. For example, experiments have shown that impurities can affect surface growth by adsorbing preferentially to step edges, ${ }^{2,3,14-16}$ where they may block the diffusion process and lead to nonsmooth growth. Certain surfactants, on the other hand, have been noticed to have an opposite effect in stabilizing smooth, layer-by-layer growth. ${ }^{17,18}$ Similarly, some impurities "poison" surfaces as they block possible reaction sites and thus hinder the reaction process, while others can promote reactions. ${ }^{14,15,19,20}$ Hydrogen, in turn, has been found to either promote or inhibit the surface diffusion of other adparticles in various systems. ${ }^{21-23}$ We can thus conclude that the role of impurities can be significant, but depends very much on the subtle interplay between the underlying surface, its structure, and the adparticles and impurities in question. Of course, this is obvious under atmospheric conditions, but what is rather surprising is that even minute impurity levels can be significant under ultrahigh-vacuum conditions. It has been noted that $\mathrm{CO}$ coverages as low as about $10^{-3}$ monolayers can affect the growth of islands during homoepitaxial growth on $\mathrm{Pt}(111),{ }^{16}$ and similar effects have been suggested for Pt dimers on $\mathrm{Pt}(110)-(1 \times 2)$ under the influence of $\mathrm{CO}$ concentrations too low to be detected by the traditional spectroscopy methods. ${ }^{24}$

From the theoretical side, the role of impurities is not well understood. Analytical theories for impurity effects on surface diffusion of single particles are still very limited, although some work has been done to clarify their role in the stability of surface growth. ${ }^{25-27}$ These analytical works have been complemented with various simulation studies of simple model systems, ${ }^{25-30}$ an approach which has also been used in studying impurity effects on the diffusion of tagged adatoms. ${ }^{31,32}$

As discussed above, however, the implications of impurities for collective diffusion have received much less attention $^{20,33}$ although many surface processes such as spreading take place over macroscopic distances, and therefore the role of surface defects and impurities is clearly significant. Two results are known in the special case of quenched, site-blocking impurities. First, Monte Carlo simulation studies have revealed ${ }^{20,33}$ that the collective diffusion coefficient $D_{C}$ of adsorbate particles can be significantly reduced from its clean surface counterpart with increasing impurity concentration. Second, in the special case of a Langmuir gas, $D_{C}$ is independent of the surface coverage with a fixed (small) impurity concentration. ${ }^{33}$ Aside from these works, the role of impurities in the collective diffusion of strongly interacting systems in equilibrium has remained an open question.

The effect of impurities on surface diffusion under nonequilibrium conditions is another aspect that is even less well understood, although processes such as surface growth and surface ordering take place in nonequilibrium. Some work has been done to clarify the role of impurities on the kinetics of domain growth, ${ }^{34,35}$ where impurities can be regarded ei- ther as a random field ${ }^{36}$ or site dilution. ${ }^{37}$ Then it has been found that the characteristic size of the domains $\bar{R}(t)$ follows a power law $\bar{R}(t) \sim t^{x}$ in time $t$ (as in the absence of impurities), but the exponent $x$ depends on the impurity concentration, ${ }^{38,39}$ which further affects the late-time behavior of the ordering process. ${ }^{40}$

We can conclude that the effect of impurities and defects on the structure and dynamics of particles on solid surfaces can be very significant. While the case of single particles is rather well understood at the moment, the situation is much more obscure in the case of collective processes. Clearly, this calls for both experimental and theoretical studies to provide one with better insight into the nature of impurities in collective surface processes.

The objective of the present work is to shed some light on these issues. We examine the influence of impurities on surface diffusion and spreading of adatoms corresponding to both equilibrium and nonequilibrium conditions. To this end, we study surface diffusion and ordering within the lattice-gas model $\mathrm{O} / \mathrm{W}(110)$, first through equilibrium simulations and then by carrying out profile spreading experiments in nonequilibrium together with the Boltzmann-Matano analysis. We examine how impurities affect the single-particle as well as collective diffusion properties of adatoms on a surface, the emphasis being on the case where there are many ordered phases at low temperatures. As a by-product, we also examine how impurities affect the ordering of the system and how these changes couple to the diffusion behavior.

We find that even minute impurity concentrations in equilibrium can lead to major changes in the diffusion coefficients. These changes are most pronounced in ordered phases and close to phase boundaries, where quenched impurities lead to a loss of the order in the system. The changes in the diffusion coefficients become more prominent as the impurity concentration is increased. For impurity concentrations of the order of a few percent of the maximum coverage, we even find changes in the phase behavior as ordered phases at large coverages essentially disappear. Further studies by the Boltzmann-Matano technique reveal that nonequilibrium effects on surface diffusion are very pronounced on clean surfaces, while on surfaces covered by impurities the role of nonequilibrium conditions is weaker. This is essentially due to the similarity between disorder as induced either by nonequilibrium conditions or by the presence of impurities on the surface. We expect that our results may have relevance to understanding some of the generic effects of impurities in systems where impurities lead to a reduction of order.

\section{THEORETICAL BACKGROUND}

\section{A. Model}

The lattice-gas Hamiltonian employed in this work is a model of the $\mathrm{O} / \mathrm{W}(110)$ adsorption system. The Hamiltonian includes contributions due to two-body and three-body interactions between oxygen adatoms, and the interaction parameters have been chosen ${ }^{41,42}$ such that the resulting phase diagram is in close agreement with the experimental observations. ${ }^{43}$ In the present study we concentrate on the 
coverage dependence of surface diffusion and ordering at a temperature of $T=500 \mathrm{~K}$ to allow direct comparison to recent equilibrium and nonequilibrium data of this system without impurities. ${ }^{41,42,44,45}$ At $T=590 \mathrm{~K}$, previous studies for a clean system in equilibrium have shown that there is a continuous phase transition around $\theta \approx 0.36$ from the lowcoverage disordered (DO) phase to the $p(2 \times 1)$ phase that is found around $\theta=1 / 2$. At higher coverages there is another transition around $\theta \approx 0.59$ from the $p(2 \times 1)$ to the $p(2 \times 2)$ phase, which in turn undergoes a transition to the disordered phase at $\theta \approx 0.78$. The locations of these phase boundaries change slightly under nonequilibrium conditions, as extracted from Boltzmann-Matano analysis. ${ }^{45}$

In the present studies, the underlying Hamiltonian for a clean $\mathrm{O} / \mathrm{W}(110)$ system is complemented to account for the role of impurities. To this end, we assume that a fixed fraction of all adsorption sites are occupied by impurities, which are described as randomly distributed quenched (immobile) adparticles. Interactions between impurities and the oxygen adatoms are described by an infinitely repulsive, on-site blocking interaction, in analogy to site dilution in lattice-gas models. Any further attractive or repulsive interactions between the impurities and oxygen adatoms are not considered here. ${ }^{46}$ The present approach should therefore be regarded as a minimal model whose aim is to examine generic effects of impurities in systems, where blocking is the main effect.

The coverage of impurity atoms is defined as $c$ $=N_{I} / N$, where $N_{I}$ is the number of impurity particles and $N$ is the total number of adsorption sites on a surface. Similarly we define the oxygen coverage $\theta=N_{\mathrm{O}} / N$ for a system of $N_{\mathrm{O}}$ oxygen atoms. To allow comparison of results with different impurity concentrations, we define

$$
\theta_{c}=\frac{\theta}{1-c},
$$

which describes the coverage of oxygen atoms on a surface where $c N$ sites are covered by immobile impurities. Our numerical data in this work have been averaged over 10-15 different randomly chosen quenched impurity configurations.

In our Monte Carlo (MC) simulations, we employ the transition dynamics algorithm ${ }^{41,44}$ (TDA) in which the diffusion events are modeled such that the thermally activated nature of a diffusion process over an adiabatic diffusion barrier has been accounted for. The use of the TDA is supported by recent molecular dynamics simulations, ${ }^{4-49}$ where it was found that the TDA is qualitatively consistent with the dynamics seen in a true microscopic model of a system consisting of interacting particles. The time is defined in terms of one Monte Carlo step (MCS), during which every particle attempts to jump once on the average. Further details and additional references of this approach can be found in Ref. 41.

\section{B. Equilibrium studies}

The equilibrium studies were made in a square system of size $L \times L$ with $L$ between 30 and 120 . Therefore the number of adsorption sites in this case is $N=L^{2}$. Periodic boundary conditions were employed in all directions. We measured the collective diffusion coefficient $D_{C}(\theta, c)$ as in Ref. 42. For a chosen $c$, we first extracted the thermodynamic factor $\xi(\theta, c)=1 / k_{B} T \theta \kappa_{T}$, which is inversely proportional to the compressibility of the adlayer, $\kappa_{T}$. This quantity is most easily extracted from grand-canonical simulations, where $N_{\mathrm{O}}$ fluctuates around $\left\langle N_{\mathrm{O}}\right\rangle$, but $N_{I}$ is fixed. Next, we determined the coefficient

$$
D_{\text {c.m. }}=\lim _{t \rightarrow \infty} \frac{1}{4 N_{\mathrm{O}} t}\left\langle\left.|| \sum_{i=1}^{N_{\mathrm{O}}}\left[\vec{r}_{i}(t)-\vec{r}_{i}(0)\right]\right|^{2}\right\rangle,
$$

which describes the center-of-mass (c.m.) motion of the whole adsorbate system in equilibrium and is obtained from canonical simulations with a fixed particle number. The c.m. diffusion coefficient is very useful in the framework of lattice-gas studies, since then it provides us with information of collective as well as single-particle motion. For lattice-gas systems, there is an exact decomposition of it as

$$
D_{\text {c.m. }}(\theta)=\frac{a^{2}}{4} \Gamma(\theta) f_{C}(\theta),
$$

where $a$ is the average jump length (usually the lattice constant) and $\Gamma$ is the average single-particle transition rate. ${ }^{13,50-52}$ The dynamical correlations between consecutive c.m. displacements are characterized by the correlation factor $f_{C}$, which for collective diffusion only rather weakly depends on ordering effects. ${ }^{42}$ Thus $D_{\text {c.m. }} \sim \Gamma$. Since the tracer diffusion coefficient $D_{T} \propto \Gamma,{ }^{51,52}$ we can use $D_{\text {c.m. }}$ to infer approximate information of single-particle diffusion rates and tracer diffusion behavior.

Having found the c.m. diffusion coefficient, the two quantities yield the collective diffusion coefficient as

$$
D_{C}=\xi D_{\text {c.m. }} .
$$

This expression clearly shows how $D_{C}$ arises from two competing factors. The thermodynamic factor plays the role of ordering via particle number fluctuations, while the centerof-mass diffusion coefficient is of dynamic nature and has its origin in the motion of individual particles.

\section{Nonequilibrium studies}

For nonequilibrium studies of surface diffusion and ordering, we consider the spreading of a coverage profile $\theta(x, t)$ in a semi-infinite system, which ranges from $-\infty$ to $+\infty$ in the $x$ direction and whose width $L_{y}$ is typically 2001000 lattice units in the $y$ direction. The exact system size used is mentioned below when relevant. Periodic boundary conditions are employed in the $y$ direction. The coverage profile is initially a step function at $x=0[\theta(x, 0)=1-c$ for $x<0$ and $\theta(x, 0)=0$ for $x>0$ ] and includes a randomly chosen impurity configuration over the whole semi-infinite slab. Thus, as the profile evolves in time $t>0$ in the $+x$ direction, the oxygen atoms have to migrate through an environment of immobile, site-blocking impurities.

To determine the collective diffusion coefficient $D_{C}(\theta)$, we use the Boltzmann-Matano (BM) method ${ }^{5,53,54}$ (as in Ref. 44), where $D_{C}(\theta)$ can be determined from scaled density profiles as 


$$
D_{C}(\theta)=\frac{1}{2 t}\left(\frac{d x}{d \theta^{\prime}}\right) \mid \int_{\theta}^{\theta} x\left(\theta^{\prime}\right) d \theta^{\prime} .
$$

The BM method has been applied to a number of experiments $^{55-61}$ and simulation studies ${ }^{44,45,62-65}$ during the last few decades.

Its popularity is largely due to a fact that it allows one to determine $D_{C}$ over a wide coverage range even from a single experiment. The disadvantage is that the coverage profiles are macroscopic (usually of the order of $1 \mu \mathrm{m}$ in the direction of spreading) (Refs. 58-61) and the experiments can last for several hours or even days. ${ }^{58,59,61}$ Thus contamination of the surface becomes a problem, and steps and other defects play a role as well. The effects of these factors are somehow included in the diffusion coefficients found by the $\mathrm{BM}$ analysis, and the problem is that there is no obvious way to decompose them.

In systems with strong interactions and ordered phases, the nonequilibrium character of spreading becomes an important issue. The BM method is based on the assumption that, in the long-time limit, the coverage profiles $\theta(x, t)$ collapse to a single scaling function when expressed as $\theta(x / \sqrt{t})$. If this condition is truly satisfied, $D_{C}(\theta)$ obtained from Eq. (5) corresponds to the actual diffusion coefficient in equilibrium. Otherwise, the effective $D_{C}(\theta)$ in Eq. (5) is a nonequilibrium quantity and depends on a time regime chosen for an analysis. For the clean O/W(110) system, Refs. 44 and 45 present a detailed study of nonequilibrium effects arising from the BM analysis. These data will be used as a reference for the present work.

\section{RESULTS}

\section{A. Impurity effects in equilibrium}

Our first task is to identify how impurities affect the diffusion behavior and ordering in equilibrium. The case of a single (tracer) particle diffusing in a lattice with moving and static background has been studied analytically, ${ }^{31,66}$ and it is known that $D_{T}$ approaches monotonically zero when the immobile impurity concentration approaches the site percolation threshold of the underlying lattice. The same result must hold for a finite concentration of tracer particles, too. Furthermore, for collective diffusion of Langmuir gas particles it has been shown that $D_{C}(\theta)$ remains constant when the impurities are immobile. ${ }^{33}$ However, for strongly interacting systems such as $\mathrm{O} / \mathrm{W}(110)$, the case is most likely very different. Therefore, we start by looking at the single-particle transition rate $\Gamma$, which is the key quantity for both tracer and collective (c.m.) diffusion (see Sec. II B).

The results shown in Fig. 1(a) for $\Gamma$ vs $\theta_{c}$ reveal that the effect of impurities is almost negligible in the disordered phase at small coverages, while in the ordered phases for $\theta_{c}>0.36$ the case is very different. Rather generally the impurities enhance the jump rate $\Gamma$ with respect to a clean surface, except at the highest coverages studied here. We expect that this behavior is coupled to the changes in spatial ordering due to the impurities, an idea which can be quantified by studying the total order parameter of the $p(2 \times 1)$ phase:

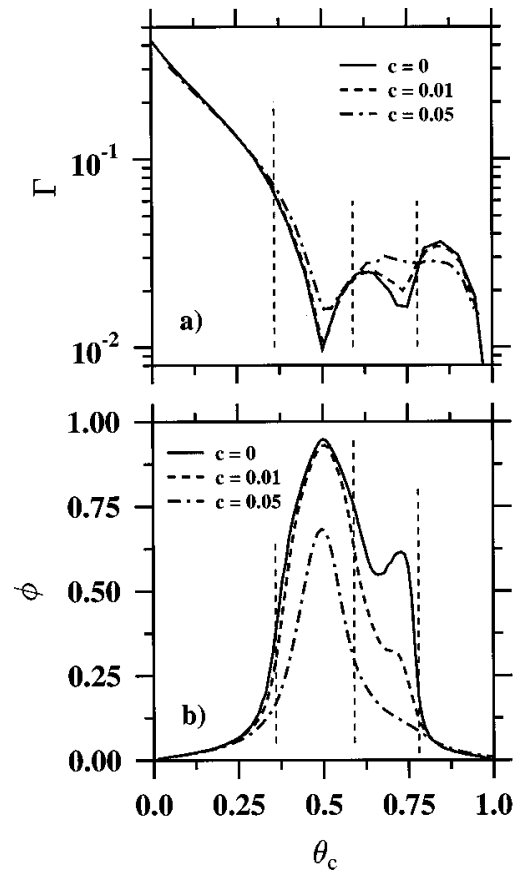

FIG. 1. (a) Equilibrium results for the average single-particle transition rate $\Gamma$ vs the coverage $\theta_{c}$ for a clean surface $(c=0)$ and for two cases including immobile impurities $(c=0.01$ and $c=0.05)$. The locations of phase boundaries for an equilibrium system at $c=0$ are shown by dashed lines. (b) Corresponding data for the order parameter $\phi$.

$$
\phi \equiv \sqrt{\phi_{2 \times 1}^{2}+\theta_{1 \times 2}^{2}} .
$$

Here $\phi_{2 \times 1}$ and $\phi_{1 \times 2}$ are the order parameter components of the degenerate $p(2 \times 1)$ and $p(1 \times 2)$ phases,

$$
\begin{aligned}
& \phi_{2 \times 1} \equiv \frac{2}{L^{2}} \sum_{i, j=1}^{L} n_{i j}(-1)^{i}, \\
& \phi_{1 \times 2} \equiv \frac{2}{L^{2}} \sum_{i, j=1}^{L} n_{i j}(-1)^{j},
\end{aligned}
$$

and are defined in terms of the occupation variables $n_{i, j}(t)$ $=0,1$ of the lattice site at $(i, j)$ in a square system of size $L \times L$. The results shown in Fig. 1(b) for $\phi$ indeed indicate that even minor impurity concentrations of the order of $1 \%$ can change the ordering properties of the adlayer at large oxygen coverages, where the density of impurities relative to vacant sites becomes more significant. For larger impurity concentrations, such as the $c=0.05$ case shown in Fig. 1(b), we find that the ordering properties change dramatically. In this case the order in the $p(2 \times 1)$ phase has weakened, although the qualitative aspects are still similar to those on a clean surface. However, for the $p(2 \times 2)$ phase the situation is much more severe. The order parameter profile $\phi\left(\theta_{c}\right)$ is almost symmetrical with respect to $\theta_{c}=1 / 2$, which means that the role of three-body correlations is now weak. This in turn implies that the $p(2 \times 2)$ phase has almost disappeared. We have not attempted a systematic finite-size scaling study to determine whether or not true long-range order is lost at higher $\theta_{c}$; in any case, $\Gamma$ is mostly sensitive to short-range order. $^{44,45}$ 


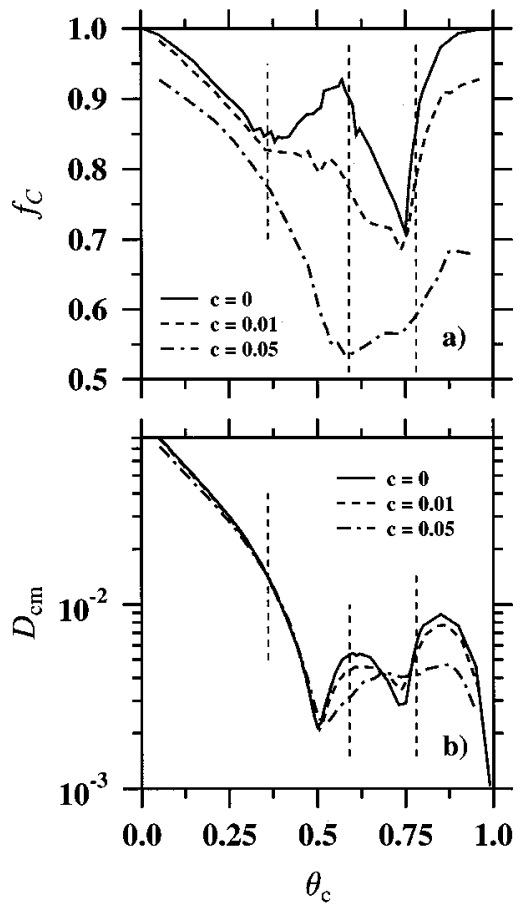

FIG. 2. (a) The dynamical correlation factor $f_{C}$ for three values of the impurity concentration $c$ in equilibrium. (b) Corresponding data for the coverage dependence of the center-of-mass diffusion coefficient $D_{\text {c.m. }}$. The locations of phase boundaries for a clean system in equilibrium are shown by dashed lines.

A comparison of Figs. 1(a) and 1(b) now reveals a direct correlation between the ordering of the system and the single-particle diffusion rate. For $c=0.01$, the average jump rate $\Gamma$ and the order parameter $\phi$ are only weakly affected by the impurities in the $p(2 \times 1)$ phase. In the $p(2 \times 2)$ phase, however, the reduced order leads to an increased diffusion rate. For $c=0.05$, the situation is rather similar in the $p(2$ $\times 1)$ phase, whereas in the $p(2 \times 2)$ phase the effects are much more dramatic. In addition to $\Gamma$ being almost constant between $0.65 \leqslant \theta_{c} \leqslant 0.85$, it has hardly any local minimum around $\theta_{c}=0.75$. This behavior is completely consistent with the shape of the order parameter profile in Fig. 1(b). We conclude that an increasing impurity concentration leads to a considerable weakening of short-range order within the ordered phases, which therefore affects the transition rates and tracer as well as collective diffusion.

Next, we focus on the correlation factor $f_{C}$ for $D_{C}$. In Fig. 2(a) we show data for $f_{C}$ vs $\theta_{c}$ without impurities ${ }^{42}$ and with $c=0.01$ and 0.05 . We can see that the effect of impurities is rather strong, with $f_{C}$ developing a broad depression at higher coverages. In Fig. 2(b) we show the corresponding data for $D_{\text {c.m. }}$. Its behavior is very similar to that of $\Gamma$ at low coverages and close to the ideal coverages of the ordered phases, namely, $1 / 2$ and $3 / 4$, where the impurities increase the diffusion rate $D_{\text {c.m. }}$ with respect to a clean system. Close to phase boundaries, on the other hand, increasing the impurity concentration leads to a reduction of $D_{\text {c.m. }}$. This behavior of $D_{\text {c.m. }}$ is due to a subtle interplay between the dynamical quantities $\Gamma$ and $f_{C}$.

We now turn our attention to the thermodynamic factor $\xi$, which is a global static quantity as it arises from average

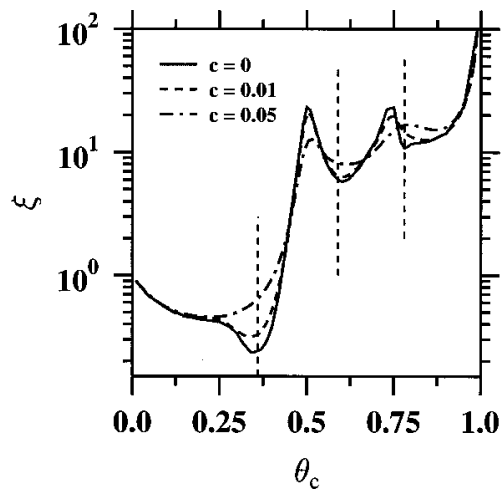

FIG. 3. Equilibrium behavior of the thermodynamic factor $\xi$ vs the coverage $\theta_{c}$. Again, the locations of phase boundaries shown by dashed lines correspond to the case $c=0$ in equilibrium.

particle number fluctuations in a whole system. As shown in Fig. 3, the prominent features in $\xi$ become smeared out with increasing $c$. The thermodynamic factor also behaves very differently from the c.m. diffusion rate. While $D_{\text {c.m. }}$ is enhanced around the ideal coverages of the ordered phases for an increasing impurity concentration, the thermodynamic factor behaves exactly in the opposite fashion. Also, while increasing impurity concentration leads $D_{\text {c.m. }}$. to decrease close to the phase boundaries between the ordered phases, the thermodynamic factor has an opposite trend. Finally, although $D_{\text {c.m. }}$ is only weakly dependent on the impurity concentration close to the boundary between the disordered and $p(2 \times 1)$ phases, the thermodynamic factor is strongly affected by the change in the impurity concentration. We have not attempted a systematic finite-size scaling of $\xi$. However, at least close to the phase boundaries of the $p(2 \times 1)$ phase we expect the finite-size effects to be rather weak as discussed in Refs. 41 and 48.

We now come to the main issue: namely, how the impurity concentration affects the collective diffusion coefficient $D_{C}$. The answer to this question lies in the discussion above, where we noted that there is an intriguing competition between $D_{\text {c.m. }}$ and $\xi$, and that their behavior is even qualitatively different close to phase boundaries and in ordered phases. This implies that a change in $D_{\text {c.m. }}$ due to an increase in $c$ is to a great extent compensated by a change of opposite direction in $\xi$. This finding is demonstrated in Fig. 4, which shows how $D_{C}$ depends on the adatom and impurity concentration in equilibrium. We find that the behavior of $D_{C}$ for an increasing impurity concentration is mostly affected at 0.3 $\leqslant \theta_{c} \leqslant 0.62$, that is, in the $p(2 \times 1)$ phase, where the behavior of $D_{C}$ is highly sensitive to the concentration of impurities. In the $p(2 \times 2)$ phase, on the other hand, changes are rather minor.

\section{B. Impurity effects in nonequilibrium}

Nonequilibrium studies of diffusion are generally rather problematic, since the diffusion coefficients are well defined only when the diffusion process can be described by the linear response theory. Recent studies have indeed shown that equilibrium definitions for diffusion coefficients do not work in nonequilibrium cases as such. ${ }^{67,68}$ Consequently, 


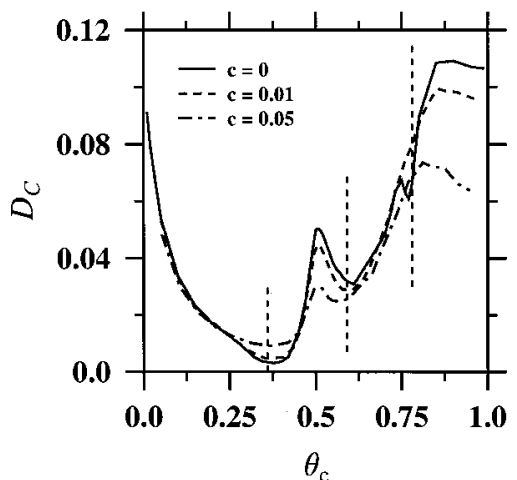

FIG. 4. The overall equilibrium behavior of the collective diffusion coefficient $D_{C}$ vs the adatom and impurity concentrations. The locations of phase boundaries for a clean system in equilibrium are shown by dashed lines.

various operational ways of defining time-dependent diffusion coefficients in terms of, e.g., particle displacement rates or mass flow ${ }^{35,67-70}$ have been suggested. Despite their operational nature, the diffusion coefficients found in this fashion are often useful since they can provide valuable information of diffusion rates under nonequilibrium conditions. However, one has to keep in mind that the rates extracted from nonequilibrium studies depend on a time regime chosen for an analysis, and the results should converge to their equilibrium limits as the system approaches equilibrium.

In the present work, we use two operational approaches for studying the diffusion behavior during a nonequilibrium process, as an initially steplike adatom layer spreads in time. First, we concentrate on the time-dependent transition rate $\Gamma(t)$ to which both $D_{C}$ and $D_{T}$ are proportional in the equilibrium limit $t \rightarrow \infty$. This quantity is computed during spread-

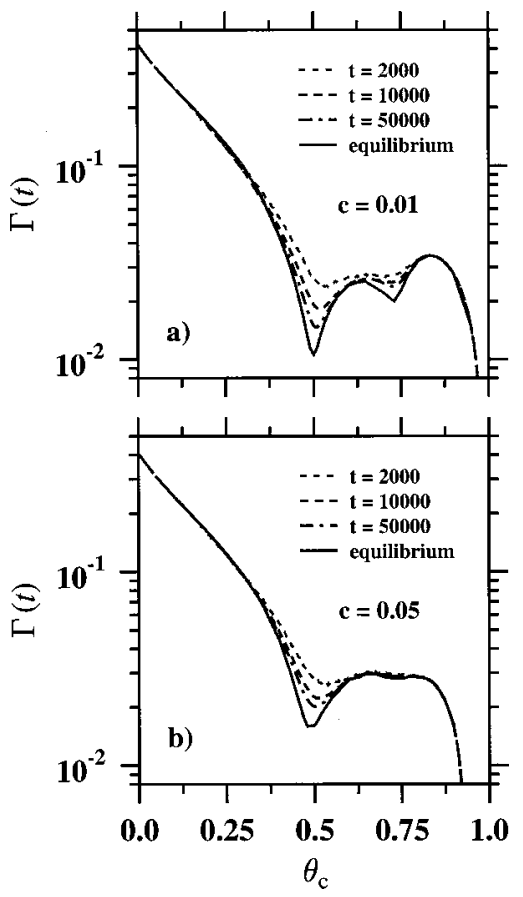

FIG. 5. Nonequilibrium results for the average single-particle transition rate $\Gamma(t)$ vs the coverage $\theta_{c}$ for two values of the impurity concentration $c$ at (a) $c=0.01$ and (b) $c=0.05$. Time is given in units of one Monte Carlo step.

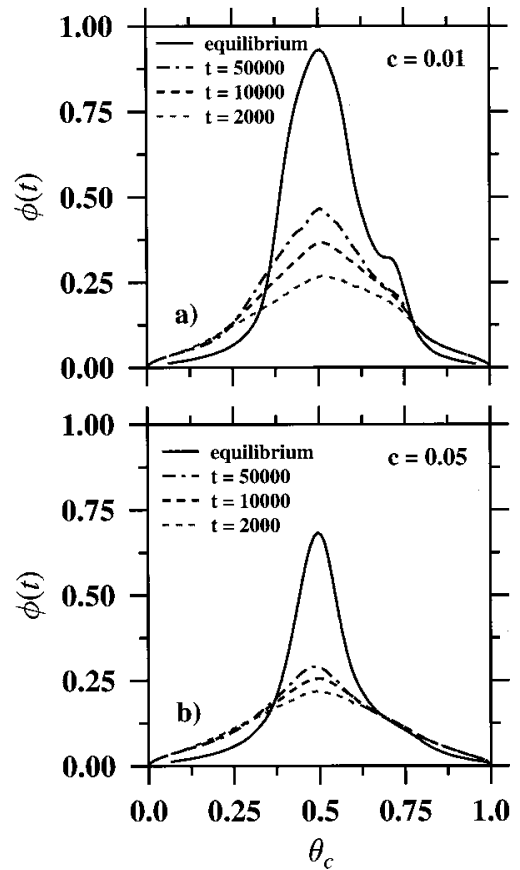

FIG. 6. (a) Nonequilibrium results for the order parameter $\phi(t)$ as in Fig. 5. Equilibrium results from Fig. 1(b) are also shown for comparison's sake. Note that the nonequilibrium results differ slightly from the equilibrium ones at small and large coverages due to a finite-size effect: The order parameter is calculated over $2 \times 200$ slabs in the $\mathrm{BM}$ simulations, while equilibrium results correspond to a system of size $60 \times 60$.

ing as explained in Refs. 44 and 45. Second, we use the BM analysis to determine the time dependence of the collective diffusion coefficient, again following Refs. 44 and 45.

Nonequilibrium results for the average transition rate $\Gamma(t)$ are shown in Figs. 5(a) and 5(b). For $c=0.01$, we find that $\Gamma(t)$ is essentially similar to its equilibrium value in the disordered phase, while in the $p(2 \times 2)$ phase there are relatively small, but distinct deviations from the equilibrium results. The largest deviations are found in the $p(2 \times 1)$ phase, where the equilibrium and nonequilibrium results differ by a factor of 2-3. For $c=0.05$, the behavior of $\Gamma(t)$ is very similar to the $c=0.01$ case, although the differences between equilibrium and nonequilibrium results are now less pronounced. In the $p(2 \times 2)$ phase, in particular, we find that the nonequilibrium behavior of $\Gamma(t)$ is essentially similar to its equilibrium limit. In both cases [Figs. 5(a) and 5(b)] the results slowly converge towards the equilibrium limit, as expected.

The order parameter profiles in Figs. 6(a) and 6(b) confirm our expectations that the system with impurities converges very slowly towards equilibrium. The slow convergence as such is not surprising, since in a recent study for a clean surface we found ${ }^{45}$ that the BM analysis gives equilibrium results only at very long times $(t \gg 250000 \mathrm{MCS})$. In the present case for $c=0.01$, there are pronounced deviations from equilibrium behavior at $t \leqslant 10000 \mathrm{MCS}$, while at later times around $t=50000 \mathrm{MCS}$ the order parameter profile begins to find its characteristic shape. Yet quantitative deviations from equilibrium remain rather large. At a larger impurity concentration of $c=0.05$, the order parameter profile approaches its equilibrium limit exceedingly slowly. 

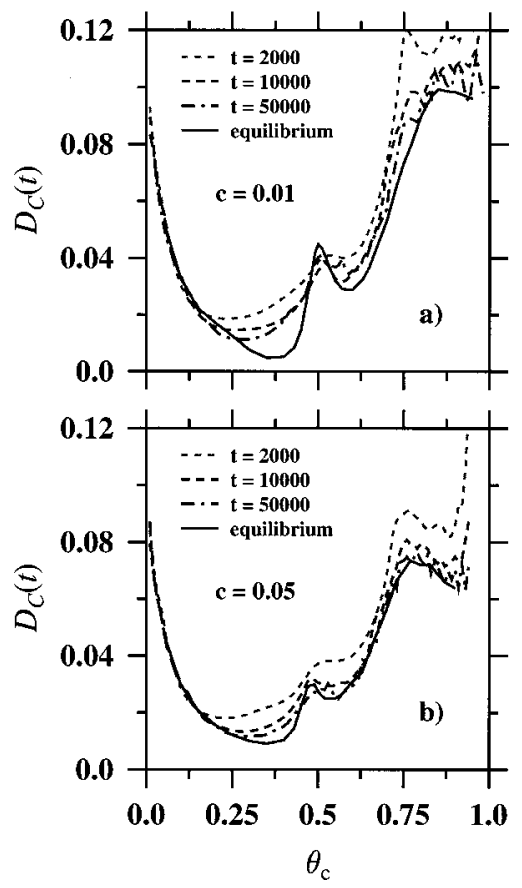

FIG. 7. Nonequilibrium behavior of the collective diffusion coefficient $D_{C}(t)$ vs the adatom and impurity concentrations with (a) $c=0.01$ and (b) $c=0.05$.

As for collective diffusion, the results in Figs. 7(a) and 7 (b) demonstrate that the nonequilibrium conditions do influence the behavior of the collective diffusion coefficient on surfaces covered by impurities. This is not too surprising in view of the previous results for the order parameter. However, it is interesting that the deviations are rather small. Except for very early times, the differences are almost negligible in the case $c=0.05$, which suggests that nonequilibrium effects in the c.m. motion and thermodynamic particle number fluctuations cancel each other in this case. For $c$ $=0.01$, the deviations are more obvious and actually rather pronounced around the $p(2 \times 1)$ phase, but still the nonequilibrium effects are rather weak. When these results are compared to those in Fig. 4, we can conclude that impurity effects on $D_{C}$ are most pronounced on a clean surface.

The results shown here suggest that nonequilibrium conditions and quenched impurities have certain similarities as regards their effects on surface diffusion. Most importantly, our results support the idea that they both reduce the ordering of the adlayer as compared to a clean system in equilibrium. We think that this finding is not specific to any particular system, but rather is of generic nature, and corresponds to a number of systems in which quenched impurities (besides blocking adsorption sites) interact relatively weakly with the adatoms migrating on a surface. For such systems, we expect that the disordering of the adatom layer due to impurities or nonequilibrium conditions leads to a number of generic effects on surface diffusion. First of all, the average transition rate is expected to be enhanced if the presence of order tends to slow down the mobilities of single particles. Second, the thermodynamic factor is smeared out and reduced close to ideal coverages of ordered phases and enhanced in the vicinity of second-order phase boundaries. This is because $\xi$ is inversely proportional to the particle number fluctuation rate $\left\langle N_{\mathrm{O}}^{2}\right\rangle-\left\langle N_{\mathrm{O}}\right\rangle^{2}$, which has a minimum in ordered phases and a maximum at continuous phase boundaries. Impurities, however, weaken the short-range order, which leads to an enhancement of particle number fluctuations within ordered phases. Close to the boundaries of continuous phase transitions, in turn, the critical behavior of the compressibility is weakened or even wiped out by the surface impurities. Consequently, particle number fluctuations are reduced as well, which eventually leads to an enhancement of the thermodynamic factor. Finally, what comes to the collective diffusion coefficient as the concentration of impurities is increased, its generic behavior depends sensitively on the relative importance of $D_{\text {c.m. }}$ and $\xi$. Consequently, there are no clear rules for saying when the c.m. diffusion coefficient or the thermodynamic factor would predominate the behavior of $D_{C}$.

\section{SUMMARY AND DISCUSSION}

Impurities are often undesired on surfaces of solid materials. Yet their presence is inevitable, since even under ultrahigh-vacuum conditions there are often tiny amounts of impurities such as hydrogen and sulfur which, among others, can affect the shape of growing islands and the diffusion characteristics of migrating adparticles. ${ }^{16,24}$ Impurities should therefore be regarded as an inherent component of any surface system. This is why both experimental and theoretical works are needed to clarify their role and effects on surface processes, such as diffusion.

In this work, we have approached this issue by examining how quenched, site-blocking impurities can influence single-particle and collective diffusion behavior at finite coverages in ordered phases. To allow a thorough analysis of impurity induced effects for both equilibrium and nonequilibrium cases, we have complemented the equilibrium studies with profile spreading simulations in nonequilibrium. All studies presented here have been done using a lattice-gas model of $\mathrm{O} / \mathrm{W}(110)$, whose equilibrium properties have been extensively characterized in the absence of impurities.

Our results show that immobile impurities can have a major effect on the ordering of adlayers at finite coverages. The effects are pronounced even at small amounts of impurities, which reduce the short-range order of an adlayer and may eventually even lead to a disappearance of long-range order. The reduction in ordering leads to profound effects on the diffusion behavior, the greatest effects being observed in equilibrium for collective diffusion. This is due to thermodynamic particle number fluctuations, which were found to be very sensitive on the ordering of the system. Under nonequilibrium conditions, we have found that the nonequilibrium diffusion results for any impurity concentration deviate clearly from the equilibrium results for a clean surface. When the nonequilibrium and equilibrium results were compared with a fixed impurity concentration level, however, the deviations were found to be relatively small.

The present results give rise to some important conclusions which we wish to discuss here briefly. First, the Boltzmann-Matano technique can be applied to transient density profiles during spreading to extract an effective, 
time-dependent collective diffusion coefficient. However, we have shown that the density profiles approach their equilibrium behavior very slowly, and therefore the profiles at early and intermediate times lead to nonequilibrium diffusion coefficients that may deviate strongly from their equilibrium counterparts. ${ }^{44,45}$ Therefore, even on clean surfaces, it is relatively difficult to find the correct equilibrium results for diffusion coefficients using the BM technique. Further problems may be faced in experiments, since it has been shown that different experimental techniques may yield different results for the diffusion coefficients ${ }^{63,71}$ and that the nonequilibrium nature of some experiments may play a role in these cases. In view of the present results, this problem is further accentuated by the presence of impurities. This should be taken into account when the experimental results are being used for a determination of phase boundaries and ordered phases.

Despite its simplicity, we feel that the present approach serves its purpose well in finding generic information of impurity-induced effects on adsorption systems. It is clear, however, that the results presented here correspond to a specific model system based on the lattice-gas description, a fact to be accounted for when the results are compared with other systems. Regarding the lattice-gas approximation, it has been demonstrated in a recent work ${ }^{72}$ that at least in the so-called high-friction limit, the many-particle diffusion properties of continuum adsorption systems are well approximated by the lattice-gas picture. A more detailed comparison to any specific system would require one to address the importance of direct attractive and repulsive interactions between impurities and adatoms. However, this may not be essential in all cases. For strongly attractive interactions, for example, it is likely that the impurities would act as traps (nucleation sites) and hence form effective impurities whose size would be a bit larger than the size of a single impurity. Then the effects due to impurities would be largely similar to those observed in this work. We feel that the case of repulsive impurityadatom interactions would be more interesting, since then the local order around a given impurity could be different from the order found elsewhere on clean parts of the surface. This issue is interesting and worth looking at, although it is beyond the scope of the present work.

It is interesting to compare our results to those presented in the classic paper by Butz and Wagner. ${ }^{55}$ In this article, they presented and discussed experimental data for collective diffusion in the $\mathrm{O} / \mathrm{W}(111)$ system. The studies were made by the Boltzmann-Matano analysis at temperatures around $760{ }^{\circ} \mathrm{C}$ (in the high-temperature disordered phase), which is somewhat higher than the temperature used in our work. However, since the transition from the $p(2 \times 1)$ phase to the disordered high-temperature phase takes place around $720 \mathrm{~K}$, we can expect the results of Ref. 55 to include ordering effects on small scales, thus allowing a qualitative comparison to some features of our data. Interestingly, Butz and Wagner found a major peak of $D_{C}(\theta)$ at $\theta \approx 0.4$ and a minor one at $\theta \approx 0.6$. Although these peaks are not exactly at the expected positions of the $p(2 \times 1)$ and $p(2 \times 2)$ phases, one should keep in mind that the studies of Ref. 55 were made over long times (5-45 min) and large distances (several mi- crometers). Thus the data of Butz and Wagner are likely influenced by impurities and steps and, possibly, even nonequilibrium conditions (see Fig. 6 in Ref. 55). This is supported by the observation that, if the concentration of ideal quenched impurities were assumed to be $c=0.2$, then the scaled oxygen coverages $\theta_{c}$ at the positions of the peaks of Ref. 55 would be 0.5 and 0.75 , corresponding to the ideal positions of the $p(2 \times 1)$ and $p(2 \times 2)$ phases. Another interesting feature of the data presented in Ref. 55 is the decrease of $D_{C}(\theta)$ at large coverages. This is in agreement with our observation that an increasing impurity concentration leads to a reduction of $D_{C}$ at large oxygen coverages (see Fig. 4). Finally, Butz and Wagner observed that the effective diffusion barrier of collective diffusion was roughly constant within the coverage range $0.4 \leqslant \theta \leqslant 0.9$. In a previous work, ${ }^{44}$ we found similar behavior for the $\mathrm{O} / \mathrm{W}(110)$ system under nonequilibrium conditions. Since the present study suggests that nonequilibrium conditions and quenched impurities have effects of the same kind on collective diffusion and ordering, the results of the present work are consistent with the findings of Ref. 55. Further experiments would be most interesting to clarify these issues.

Our final point concerns the range of interactions and how it may affect the "critical" impurity concentration above which any deviations from the behavior corresponding to a clean surface might be observed. As pointed out above, the interaction range between impurities and adatoms in the present model is very short. In many systems studied in experiments, however, the interaction range can be much larger due to (say) dipole-dipole interactions or due to interactions mediated by the substrate. This may have a major effect on how readily the role of impurities comes up. We expect that the larger is the interaction range, the smaller is the "critical" impurity concentration. Therefore, although here we have noticed major effects on diffusion and ordering for impurity concentrations of the order of a few percent of the monolayer, in systems with long-range interactions such effects are likely at much smaller impurity concentrations. Recent experiments and model studies report this idea indirectly. ${ }^{16,24,73-76}$

We close this work by a brief note about actual conditions where surfaces are usually studied. Surfaces are typically nothing but ideal. They contain various kinds of defects and contaminants that change the characteristics of surfaces and consequently their diffusion properties. Yet most theoretical and numerical approaches done by far have focused on understanding the case of an ideal surface. However, we are now at a stage where many of the prominent features of diffusion on idealized surfaces are well understood. Consequently, it would be worthwhile to direct more and more effort to examine the properties of surfaces under more realistic conditions. The case where the role of impurities and other surface active particles is accounted for is one, but not the only one, of the many situations. Further problems remain, and we are looking forward to future work that addresses these complicated issues. 


\section{ACKNOWLEDGMENTS}

This work has been supported in part by the Academy of Finland through its Center of Excellence Program and by a grant from the European Union (I.V.).

${ }^{1}$ H. Lüth, Surfaces and Interfaces of Solid Materials, 3rd ed. (SpringerVerlag, Berlin, 1998).

${ }^{2}$ Surface Diffusion: Atomistic and Collective Processes, edited by M. C. Tringides (Plenum, New York, 1997).

${ }^{3}$ J. V. Barth, Surf. Sci. Rep. 40, 75 (2000).

${ }^{4}$ M. Giesen, Prog. Surf. Sci. 68, 1 (2001).

${ }^{5}$ R. Gomer, Rep. Prog. Phys. 53, 917 (1990)

${ }^{6}$ A. G. Naumovets and Yu. S. Vedula, Surf. Sci. Rep. 4, 365 (1985).

${ }^{7}$ G. L. Kellogg, Surf. Sci. Rep. 21, 1 (1994).

${ }^{8}$ H. Brune, Surf. Sci. Rep. 31, 121 (1998).

${ }^{9}$ T. R. Linderoth, S. Horch, L. Petersen, S. Helveg, E. Laegsgaard, I. Stensgaard, and F. Besenbacher, Phys. Rev. Lett. 82, 1494 (1999).

${ }^{10}$ R. van Gastel, E. Sonfai, S. B. van Albada, W. van Saarloos, and J. W. M. Frenken, Phys. Rev. Lett. 86, 1562 (2001).

${ }^{11}$ T. Ala-Nissila, R. Ferrando, and S. C. Ying, Adv. Phys. 51, 949 (2002).

${ }^{12}$ T. Ala-Nissila and S. C. Ying, Surf. Sci. 39, 227 (1992).

${ }^{13}$ A. Danani, R. Ferrando, E. Scalas, and M. Torri, Int. J. Mod. Phys. B 11, 2217 (1997).

${ }^{14}$ X.-D. Xiao, Y. Xie, C. Jacobsen, H. Galloway, M. Salmeron, and Y. R. Shen, Phys. Rev. Lett. 74, 3860 (1995).

${ }^{15}$ X.-D. Xiao, Y. Xie, C. Jacobsen, and Y. R. Shen, Phys. Rev. B 56, 12529 (1997).

${ }^{16}$ M. Kalff, G. Comsa, and Th. Michely, Phys. Rev. Lett. 81, 1255 (1998).

${ }^{17}$ S. Esch, M. Hohage, T. Michely, and G. Comsa, Phys. Rev. Lett. 72, 518 (1994).

${ }^{18}$ B. Voigtländer, A. Zinner, T. Weber, and H. P. Bonzel, Phys. Rev. B 51, 7583 (1995).

${ }^{19}$ C. H. Mak, J. L. Brand, A. A. Deckert, and S. M. George, J. Chem. Phys. 85, 1676 (1986).

${ }^{20}$ J. L. Brand, A. A. Deckert, and S. M. George, Surf. Sci. 194, 457 (1988).

${ }^{21}$ G. L. Kellogg, Phys. Rev. B 55, 7206 (1997).

${ }^{22}$ J. Nara, T. Sasaki, and T. Ohno, Phys. Rev. Lett. 79, 4421 (1997).

${ }^{23}$ S. Horch, H. T. Lorensen, S. Helveg, E. Laegsgaard, I. Stensgaard, K. W. Jacobsen, J. K. Nørskov, and F. Besenbacher, Nature (London) 398, 134 (1999).

${ }^{24}$ T. R. Linderoth, S. Horch, L. Petersen, S. Helveg, M. Schonning, E. Laegsgaard, I. Stensgaard, and F. Besenbacher, Phys. Rev. B 61, R2448 (2000).

${ }^{25}$ L. A. N. Amaral and J. Krug, Phys. Rev. E 55, 7785 (1997).

${ }^{26}$ Y. Yoshioka, T. Matsui, M. Kasuga, and T. Irisawa, J. Cryst. Growth 198/199, 71 (1999).

${ }^{27}$ M. Kotrla, J. Krug, and P. Šmilauer, Surf. Sci. 482-485, 840 (2001).

${ }^{28}$ W. J. P. van Enckevort and A. C. J. F. van der Berg, J. Cryst. Growth 441-455, 183 (1998).

${ }^{29}$ K. Haug and N.-K. N. Do, Phys. Rev. B 60, 11095 (1999).

${ }^{30}$ L. Gómez and J. Ferrón, Phys. Rev. B 64, 033409 (2001).

${ }^{31}$ J. W. Haus and K. W. Kehr, Phys. Rep. 150, 263 (1987).

${ }^{32}$ S. M. Lee, Y. H. Lee, and N. Kim, Surf. Sci. 470, 89 (2000)

${ }^{33}$ C. H. Mak, H. C. Andersen, and S. M. George, J. Chem. Phys. 88, 4052 (1988).

${ }^{34}$ Kinetics of Ordering and Growth at Surfaces, edited by M. G. Lagally (Plenum, New York, 1990).

${ }^{35}$ M. C. Tringides, in The Chemical Physics of Solid Surfaces and Heterogeneous Catalysis: Phase Transitions and Restructuring of Metal Surfaces, edited by D. A. King and D. P. Woodruff (Elsevier, Amsterdam, 1994), Vol. 7, Chap. 6.

${ }^{36}$ M. Grant and J. D. Gunton, Phys. Rev. B 29, 1521 (1984).

${ }^{37}$ D. Chowdhury, M. Grant, and J. D. Gunton, Phys. Rev. B 35, 6792 (1987).

${ }^{38}$ J.-K. Zuo, G.-C. Wang, and T.-M. Lu, Phys. Rev. Lett. 60, 1053 (1988).

${ }^{39}$ J.-K. Zuo, G.-C. Wang, and T.-M. Lu, Phys. Rev. B 40, 524 (1989).

${ }^{40}$ D. J. Srolovitz and G. N. Hassold, Phys. Rev. B 35, 6902 (1987).

${ }^{41}$ I. Vattulainen, J. Merikoski, T. Ala-Nissila, and S. C. Ying, Phys. Rev. B 57, 1896 (1998).

${ }^{42}$ I. Vattulainen, S. C. Ying, T. Ala-Nissila, and J. Merikoski, Phys. Rev. B 59, 7697 (1999).
${ }^{43}$ G.-C. Wang, T.-M. Lu, and M. G. Lagally, J. Chem. Phys. 69, 479 (1978); C. R. Brundle and J. Q. Broughton, in The Chemical Physics of Solid Surfaces and Heterogeneous Catalysis: Chemisorption Systems, edited by D. A. King and D. P. Woodruff (Elsevier, Amsterdam, 1990), Vol. 3A, Chap. 3; K. E. Johnson, R. J. Wilson, and S. Chiang, Phys. Rev. Lett. 71, 1055 (1993)

${ }^{44}$ P. Nikunen, I. Vattulainen, and T. Ala-Nissila, Surf. Sci. 447, L162 (2000).

${ }^{45}$ P. Nikunen, I. Vattulainen, and T. Ala-Nissila, J. Chem. Phys. 114, 6335 (2001)

${ }^{46}$ Including further repulsive or attractive impurity-oxygen interactions into the model would be straightforward, but not justified. First, we wish to study generic features of impurities in surface systems rather than focus on any impurity in particular. Second, even if some particular atom such as sulfur were chosen to act as an impurity, we lack any knowledge of its interactions with oxygen on W(110). Finding such information would require very careful experiments or first-principles calculations, which under present circumstances are not available.

${ }^{47}$ I. Vattulainen, S. C. Ying, T. Ala-Nissila, and J. Merikoski, J. Chem. Phys. 111, 11232 (1999)

${ }^{48}$ I. Vattulainen, J. Merikoski, T. Ala-Nissila, and S. C. Ying, Phys. Rev. Lett. 80, 5456 (1998)

${ }^{49}$ I. Vattulainen, J. Merikoski, S. C. Ying, and T. Ala-Nissila, Europhys. Lett. 51, 361 (2000).

${ }^{50}$ D. A. Reed and G. Ehrlich, Surf. Sci. 102, 588 (1981).

${ }^{51}$ T. Hjelt, I. Vattulainen, J. Merikoski, T. Ala-Nissila, and S. C. Ying, Surf. Sci. 380, L501 (1997)

${ }^{52}$ T. Hjelt, I. Vattulainen, J. Merikoski, T. Ala-Nissila, and S. C. Ying, Surf. Sci. 402-404, 253 (1998)

${ }^{53}$ C. Matano, Jpn. J. Phys. 8, 109 (1933)

${ }^{54}$ J. Crank, The Mathematics of Diffusion (Oxford University Press, Oxford, 1995).

${ }^{55}$ R. Butz and H. Wagner, Surf. Sci. 63, 448 (1977).

${ }^{56}$ E. Suliga and M. Henzler, J. Phys. C 16, 1543 (1983).

${ }^{57}$ A. G. Naumovets, M. V. Paliy, and Yu. S. Vedula, Phys. Rev. Lett. 71, 105 (1993)

${ }^{58}$ A. T. Loburets, A. G. Naumovets, N. B. Senenko, and Yu. S. Vedula, Z. Phys. Chem. (Münich) 202, 75 (1997).

${ }^{59}$ A. T. Loburets, A. G. Naumovets, and Yu. S. Vedula, Surf. Sci. 399, 297 (1998).

${ }^{60}$ M. Šnábl, M. Ondřejček, V. Cháb, Z. Chvoj, W. Stenzel, H. Conrad, and A. M. Bradshaw, J. Chem. Phys. 108, 4212 (1998).

${ }^{61}$ S. Tsukawaki, Y. Hatano, K. Hashizume, and M. Sugisaki, Surf. Sci. 476, 115 (2001).

${ }^{62}$ M. Bowker and D. A. King, Surf. Sci. 71, 583 (1978).

${ }^{63}$ J. Haas, K. R. Roos, A. Jesina, and M. C. Tringides, Surf. Sci. 307-309, 465 (1994).

${ }^{64}$ J. Sanchez, D. P. Quinn, and M. C. Tringides, Surf. Sci. 391, 101 (1997).

${ }^{65}$ M. A. Zaluska-Kotur, S. Krukowski, Z. Romanowski, and L. A. Turski, Surf. Sci. 457, 357 (2000).

${ }^{66}$ R. A. Tahir-Kheli, Phys. Rev. B 28, 3049 (1983).

${ }^{67}$ I. Vattulainen, J. Merikoski, T. Ala-Nissila, and S. C. Ying, Surf. Sci. 366, L697 (1996)

${ }^{68}$ E. Arapaki, P. Argyrakis, and M. C. Tringides, Phys. Rev. B 62, 8286 (2000).

${ }^{69}$ M. C. Tringides, P. K. Wu, and M. G. Lagally, Phys. Rev. Lett. 59, 315 (1987).

${ }^{70}$ P. K. Wu, M. C. Tringides, and M. G. Lagally, Phys. Rev. B 39, 7595 (1989).

${ }^{71}$ M. C. Tringides and R. Gomer, Surf. Sci. 265, 283 (1992).

${ }^{72}$ J. M. Lahtinen, M. Mašín, T. Laurila, T. Ala-Nissila, and Z. Chvoj, J. Chem. Phys. 116, 7666 (2002).

${ }^{73}$ L. Österlund, M. Ф. Pedersen, I. Stensgaard, E. Laegsgaard, and F. Besenbacher, Phys. Rev. Lett. 83, 4812 (1999).

${ }^{74}$ K. Fichthorn and M. Scheffler, Phys. Rev. Lett. 84, 5371 (2000).

${ }^{75}$ A. Bogicevic, S. Ovesson, P. Hyldgaard, B. I. Lundqvist, H. Brune, and D. R. Jennison, Phys. Rev. Lett. 85, 1910 (2000).

${ }^{76}$ J. Repp, F. Moresco, G. Meyer, and K.-H. Rieder, Phys. Rev. Lett. 85, $2981(2000)$ 